\title{
Aux origines de la loi de Darcy (1856)
}

The origins of Darcy's law (1856)

\section{Martin Zerner}

\section{(2) OpenEdition}

\section{Journals}

Édition électronique

URL : http://journals.openedition.org/dht/1625

DOI : $10.4000 /$ dht. 1625

ISSN : 1775-4194

Éditeur :

Centre d'histoire des techniques et de l'environnement du Cnam (CDHTE-Cnam), Société des élèves du CDHTE-Cnam

\section{Édition imprimée}

Date de publication : 1 décembre 2011

Pagination : $29-40$

ISBN : 978-2-9530779-7-1

ISSN : 0417-8726

\section{Référence électronique}

Martin Zerner, «Aux origines de la loi de Darcy (1856) », Documents pour l'histoire des techniques [En ligne], 20 | 2e semestre 2011, mis en ligne le 14 septembre 2012, consulté le 08 septembre 2020. URL : http://journals.openedition.org/dht/1625; DOI : https://doi.org/10.4000/dht.1625 


\title{
Aux origines de la loi de Darcy (1856)
}

\author{
Martin Zerner \\ Équipe REHSEIS \\ UMR 7219 SPHERE (CNRS et Université Paris Diderot-Paris VII)
}

\begin{abstract}
Résumé
L'article analyse les expériences qui ont conduit Darcy à l'énoncé de sa loi de la filtration dans le contexte de ses autres travaux sur l'hydraulique. Le lien avec ses résultats sur les écoulements lents dans les tuyaux est clarifié. Quoiqu'il ne l'ait certainement pas reconnu aussi clairement que Hagen dans des travaux plus anciens, Darcy semble avoir compris qu'il y avait là ce que nous appelons un changement de régime. Une autre piste qui l'a conduit à la loi de la filtration est l'étude des puits artésiens. II est difficile d'être plus précis ici, parce que la chronologie est incertaine. En tout cas, il a déduit la relation entre le débit et la hauteur de l'ouverture de la loi de la filtration, il l'a aussi vérifiée expérimentalement, et il a considéré le résultat positif de l'expérience comme une confirmation de cette loi.
\end{abstract}

Résumés et mots clés en anglais sont regroupés en fin de volume, accompagnés des mots clés français

Q i visite Dijon ne peut manquer de remarquer immense place Darcy proche de la gare et flanquée du jardin du même nom. Ce n'est que justice, car l'ingénieur des Ponts et Chaussées Henry Darcy a beaucoup fait pour la prospérité de la ville. Le plus important est son action pour faire passer par Dijon le chemin de fer de Paris à Lyon, un facteur décisif pour transformer une petite ville bourguignonne en métropole régionale. Nous n'en parlerons pas ici. Les archives municipales de Dijon ont organisé en 2003 une exposition à l'occasion du bicentenaire de la naissance de Darcy. On trouvera des études et des bibliographies dans la brochure qui a accompagné cette exposition'. L'article d'Eliane Lochot, conservatrice aux archives municipales, contient une biographie et une liste des sources complète 2 . On y trouvera la référence à d'autres biographies. Nous ne donnerons plus loin que les éléments biographiques utiles à la compréhension de son œuvre d'hydraulicien.

1 Éliane Lochot et Brice Grivot-Boone dir., Henry Darcy, le bicentenaire, 1803 2003, Dijon, Archives municipales de Dijon, 2003. 2 Éliane Lochot, "L'homme et ses réalisations », dans É. Lochot et B. Grivot-Boone dir., Henry Darcy, op. cit., pp. 7-21.
La ville de Dijon doit aussi à Darcy, et cela nous rapproche de l'objet du présent article, son système d'alimentation en eau. L'ingénieur a publié un livre monumental exposant les études qu'il a faites à cet effet, Les fontaines publiques de la ville de Dijon ${ }^{3}$. C'est un ouvrage souvent cité, et sûrement beaucoup moins lu, par les ingénieurs d'aujourd'hui parce qu'on y trouve exposée pour la première fois la loi de Darcy. Celle-ci régit l'écoulement de l'eau à travers le sable, et plus généralement celui d'un fluide à travers un milieu poreux. Elle possède de multiples applications techniques, en hydraulique bien sûr, mais aussi pour l'extraction du pétrole, l'enrichissement de l'uranium et j'en oublie, sans parler de celles que je ne connais pas.

L'histoire de l'hydrodynamique et de l'hydraulique a été relativement peu étudiée. Jusqu'à la parution du livre de Darrigol ${ }^{4}$, en dehors d'un certain nombre d'articles plus ou moins spécialisés et d'ouvrages collectifs $^{5}$, on ne disposait que de peu d'ouvrages

3 Henry Darcy, Les Fontaines publiques de la Ville de Dijon, Paris, Victor Dalmont, 1856.

4 Olivier Darrigol, Worlds of flow : A history of hydrodynamics from the Bernoulli's to Prandtl, Oxford University Press, 2005.

5 Voir, par exemple, Günther Garbrecht dir., Hydraulics and hy- 
d'ensemble, dont le petit livre « classique » de Hunter Rouse et Simon Ince ${ }^{6}$, en fait une suite d'indications certes précieuses mais sommaires. Au sein de cette historiographie plutôt maigre, la mécanique des fluides en milieu poreux faisait encore récemment figure de parent pauvre. Voici, in extenso, ce que Rouse et Ince disent de la loi de Darcy : « Le résultat pertinent des études de Darcy sur la filtration - que la perte de charge était proportionnelle au débit, et non, comme on le pensait communément, à sa racine carrée - était contenu dans son traité publié en 1856 à Paris, Les Fontaines publiques de la Ville de Dijon 17 . Ils ajoutent seulement quelques mots sur les applications qu'en a faites Jules Dupuit (1804-1866) ${ }^{8}$.

La situation a changé avec le deuxième centenaire de la naissance de Darcy (2003) et le cent-cinquantenaire de sa loi (2006). Nous avons déjà parlé de l'exposition des archives municipales de Dijon et de la brochure qui l'a accompagnée et de l'article d'Éliane Lochot. Un colloque a été organisé par l'ASCE (American Society of Civil Engineers) à l'occasion du deuxième centenaire de la naissance; les actes en sont publiés?. Des chercheurs de la Oklahoma State University ont mis en ligne un certain nombre d'articles et la note de Darcy dans laquelle il établit la loi qui porte son nom ${ }^{10}$. Une traduction du livre sur les fontaines de Dijon a été publiée"l (Darcy 2004). A l'occasion du cent cinquantième anniversaire de la publication des Fontaines publiques de la Ville de Dijon une journée d'un colloque sur la gestion des grands aquifères tenu à Dijon a été consacrée à Darcy. Deux articles des actes de ce colloque intéressent notre sujet : I'un décrit le livre et l'autre donne, outre une biographie de Darcy, une description de son œuvre scientifique ${ }^{12}$. Un

draulic research. A historical review, Rotterdam, Boston, A.A., 1987. 6 Hunter Rouse et Simon Ince, History of Hydraulics, lowa City, lowa Institute of hydraulic Research, 1957 ; 2 $2^{\text {ème }}$ édition Dover 1963. 7 « The pertinent result of Darcy's filtration studies - that the loss of head was proportional to the rate of flow rather than, as populary [sic] supposed, to its square root - was contained in his 1856 Paris treatise Les Fontaines publiques de la Ville de Dijon » (H. Rouse et S. Ince, History of hydraulics, op. cit., p. 171). 8 lbid., pp. 121-172.

9 Glenn O. Brown, Günter Garbrecht et Willi Hager dir., Henry P. G. Darcy and other Pioneers in Hydraulics, Reston, ASCE, 2003 $10 \mathrm{http} / / /$ biosystems.okstate.edu/darcy/

11 Henry Darcy, The public Fountains of the City of Dijon, Dubuque (lowa), Kendall-Hunt Publishing Company, 2004, trad. P. Bobeck.

12 Patricia Bobeck, "Henry Darcy's Public Fountains of the City of Dijon ", dans Laurence Chery \& Ghislain de Marsily dir., Aquifer Systems Management: Darcy's Legacy article récent de Ritzi et Bobeck contient une lecture comparative des travaux de Darcy et Dupuit sur I'hydraulique des milieux poreux ${ }^{13}$. Une version partielle et préliminaire du présent article a été présentée aux journées InterIREM "Sur les liens entre mathématiques et sciences physiques " de Dijon. ${ }^{14}$

\section{Une biographie orientée}

Henry Darcy est né en 1803 à Dijon où son père était receveur des impôts. II entre à Polytechnique en 1821, à l'école des Ponts et Chaussées en 1823. À sa sortie, il est affecté au département du Jura mais rapidement transféré à Dijon, à la demande du préfet de la Côte d'Or. On notera que cela se produit sous la Restauration. La tâche confiée à Darcy était une étude préliminaire d'un projet d'adduction d'eau pour la ville. Son travail aboutit en 1834 à un rapport à la municipalité dans lequel il propose un plan détaillé basé sur l'adduction de l'eau de la source du Rosoir située à douze kilomètres de la ville. Le plan rapidement approuvé par le conseil municipal, les travaux commencent en 1839 (il fallait l'approbation du projet par le gouvernement, le temps de réaliser des expropriations et la passation des marchés). L'eau du Rosoir commence à arriver à Dijon l'année suivante et les travaux sont achevés en 1844.

Darcy est obligé de quitter Dijon après la révolution de 1848 et affecté à Bourges, parce qu'il était orléaniste et, en tant que conseiller municipal, participait à la vie politique locale. Le 16 juin 1848 il est nommé à Paris comme directeur du service de l'eau et du pavage. Il en profite pour mettre en chantier la série d'expériences sur le mouvement de l'eau dans les tuyaux dont nous parlerons plus loin. Le 30 avril 1850 il est promu inspecteur des Ponts et Chaussées. C'est Dupuit qui le remplace. Il lui donne la possibilité de poursuivre les mesures qui viennent à peine de commencer.

in a World of Impending Water Shortage, Reading, International Association of Hydrogeologists, 2007, pp. 4-9.

Craig Simmons, « Henry Darcy (1803-1858) : immortalised by his scientific Legacy $»$, ibidem pp. 10-26.

13 Robert Ritzi et Patricia Bobeck, "Comprehensive principles of quantitative hydrogeology" established by Darcy (1856) and Dupuit (1857), Water Resources Research, vol. 44, 2008, pp. 1-14. 14 Martin Zerner, "Darcy et les fontaines de la ville de Dijon », in Sur les liens entre mathématiques et sciences physiques, IREM de Dijon, 2007, p. 97-103. 
Darcy a alors à superviser une circonscription située dans le Massif central, puis une autre dans les pays de la Loire. II retourne à Dijon en 1855, mais la même année, il est déchargé de service pour raison de santé. II garde cependant la possibilité de poursuivre son travail de recherche. II bénéficie pour cela de la collaboration d'autres ingénieurs des Ponts et Chaussées. En particulier, Henry Bazin (1829-1917) l'assistera dans ses expériences sur l'écoulement de l'eau dans les canaux ${ }^{15}$. II les poursuivra après la mort de Darcy en 1858.

Darcy publie en 1856 Les Fontaines publiques de la Ville de Dijon. Nous reparlerons de ce livre monumental souvent cité parce qu'on y trouve en quelques pages la loi qui porte maintenant le nom de Darcy. L'année suivante, Darcy publie Recherches expérimentales relatives au Mouvement de l'Eau dans les Tuyaux. II mourut le 2 janvier 1858. Une note "Relative à quelques modifications à introduire dans le tube de Pitot " paraît après sa mort'16. En 1865, Bazin publie Recherches hydrauliques entreprises par M. H. Darcy ${ }^{17}$.

Darcy laisse donc une œuvre double d'ingénieur et de scientifique, cette dernière exclusivement dans le domaine de l'hydraulique ${ }^{18}$. Le chef-d'œuvre de l'ingénieur est l'adduction d'eau de Dijon. Elle a marqué la physionomie de la ville avec en particulier l'installation d'une borne-fontaine tous les cent mètres et les jets d'eau de la Porte SaintPierre (aujourd'hui, Place Wilson). Le débit pouvait atteindre au besoin plus de $11.000 \mathrm{~m}^{3}$ par jour. L'eau était gratuite aux bornes-fontaines. Darcy y tenait beaucoup contrairement à un autre ingénieur des Ponts et Chaussées. II s'agit de Dupuit auquel nous avons déjà fait allusion et que nous retrouverons. ॥ est beaucoup plus connu comme économiste que

15 Ils utilisent une dérivation du canal de Bourgogne en cours de construction. Plus tard, Bazin fera d'autres expériences à la demande de Boussinesq. C'est l'occasion de signaler l'intérêt de leur correspondance conservée à la Bibliothèque de I'Institut de France (MS4228).

16 Le tube de Pitot est un instrument qui permet de mesurer la vitesse d'un liquide loin de la surface.

17 Henry Darcy et Henry Bazin, Recherches hydrauliques entreprises par M. H. Darcy, Paris, Imprimerie Nationale, 1865.

18 II ne sera pas le seul polytechnicien de la première moitié du XIXe siècle à produire une telle œuvre double. Voir entre autres : Bruno Belhoste et Konstantinos Chatzis, « From technical corps to technocratic power : French state engineers and their professional universe in the first half of the $19^{\text {th }}$ century "I, History and Technology, vol. 23, n³, 2007, pp. 209-225. comme ingénieur'19. L'adduction d'eau du Rosoir est au demeurant toujours en service aujourd'hui.

Les principales contributions scientifiques de Darcy ont déjà été mentionnées. La loi de Darcy est la plus connue aujourd'hui. Elle semble être la plus originale et, comme il a été dit, elle est à la base des calculs des ingénieurs dans de multiples domaines d'application. Si à première vue il paraît facile de séparer l'œuvre de l'ingénieur de celle du scientifique, il ne faut pas s'y tromper, elles sont fortement liées. Ce point se rattache à l'étude du milieu des ingénieurs des Ponts et Chaussées ${ }^{20}$. Certains d'entre eux ont apporté des contributions scientifiques dont l'importance est bien connue, on pense en particulier à Navier (1785-1836) et Barré de Saint-Venant (1797-1886). Mais ceux-ci, contrairement

19 II y a de nombreux travaux sur Dupuit économiste. Citons, entre autres, la conférence d'Ekelund et Hébert « Jules Dupuit, ingénieur et économiste "): http://driout.club.fr/ Jules_Dupuit.html, et, surtout, la réédition critique de son œuvre économique : Yves Breton et Gérard Klotz, Jules Dupuit, oeuvres économiques complètes (établies et présentées par Yves Breton et Gérard Klotz), 2 volumes, Paris, Economica, 2009. Sur la question du prix de l'eau chez Dupuit : Konstantinos Chatzis et Olivier Coutard, « Dupuit à propos du mode de distribution et du prix de vente des eaux aux particuliers " dans Jean-Pascal Simonin et François Vatin éd., L'OEuvre multiple de Jules Dupuit (1804-1866). Calcul d'Ingénieur, Analyse économique et Pensée sociale, Presses de l'Université d'Angers, 2002, pp. 71-88. Darcy a exprimé sa position dans son livre sur les fontaines de Dijon. Je n'ai pas connaissance d'une discussion directe entre les deux hommes. Sur Dupuit hydraulicien : H. Rouse et S., Ince, History of Hydraulics, op.cit., p. 167-168 et 171-172; Glenn O. Brown, « Jules Dupuit's contribution in water resources ॥, dans Jerry R. Rogers, Jürgen Garbrecht et Willi Hager dir., Water resources and environmental history, Reston, American Society of Civil Engineers, 2004 ; Jürgen Hager, « Jules Dupuit - eminent hydraulic Engineer II, J. Hydr. Engrg 130, 2004, pp. 843-848 ; Robert Ritzi Jr. et Patricia Bobeck, Comprehensive principles of quantitative hydrogeology established by Darcy (1856) and Dupuit (1857), Water Resources Research, vol. 44, 2008, pp. 1-14.

20 Bruno Belhoste, « Un modèle à l'épreuve. L'École Polytechnique de 1794 au second Empire "1, dans Bruno Belhoste, Amy Dahan et Antoine Picon dir., La formation polytechnicienne. 1794-1994, Paris, Dunod, 1994, pp. 9-30 ; Konstantinos Chatzis, "Les conceptions de Barré de Saint Venant (1797-1886) en matière de théorie de la connaissance $"$, Bulletin de la Société archéologique du Vendômois, 2004, pp. 70-78; Konstantinos Chatzis, « Jules Dupuit, ingénieur des ponts et chaussées ॥, dans Y. Breton et G. Klotz), op. cit. pp. 615-692 ; Antoine Picon L'invention de l'Ingénieur moderne: l'École des Ponts et Chaussées. 1747-1851, Paris, Presses de l'École Nationale des Ponts et Chaussées, 1992. 
à Darcy, sont plus des enseignants et des chercheurs que des hommes de terrain (Saint-Venant contre son gré). Chez beaucoup d'autres ingénieurs des Ponts et Chaussées, l'empirisme, exprimé ou non, régnait en maître ${ }^{21}$. Saint-Venant s'est plaint amèrement de l'empirisme de ses collègues ingénieurs. II a expliqué cette opposition dans divers écrits publiés ou inédits ${ }^{22}$. Pour lui, l'emploi d'une formule empirique, motivé sur ce qu'on peut la faire concorder avec quelques faits isolés, ne fera que tromper et empêcher d'y substituer une formule vraie. Nous verrons que ce point de vue est confirmé par l'étude de la naissance de la loi de Darcy. II serait intéressant de connaître les éventuelles relations entre ce dernier et Saint-Venant qui n'ont pas été étudiées à notre connaissance.

\section{Un aperçu du livre sur les fontaines de Dijon}

Ce livre aborde les sujets les plus divers ; c'est un véritable monde. Commençons sèchement : 647 pages auxquelles il faut ajouter 28 planches hors texte texte de $35,4 \mathrm{~cm}$ sur $57,7 \mathrm{~cm}$ qui ne se trouvent pas, comme il était habituel, à la fin de l'ouvrage mais dans un album à part. La table des matières à elle seule occupe sept pages; il est vrai qu'elle est très détaillée.La page de titre porte les sous-titres suivants : "Exposition et application des principes à suivre et des formules à employer dans les questions de distribution d'eau; ouvrage terminé par un appendice relatif aux fournitures d'eau de plusieurs villes, au filtrage des eaux et à la fabrication des tuyaux de fonte, de plomb, de tôle et de bitume ».

Une première partie est consacrée à expliquer le choix de la source du Rosoir. Plus précisément, c'est l'objet du chapitre III qui occupe environ 120 pages. II est précédé de deux chapitres historiques (une cinquantaine de pages à eux deux); à part une citation de Grégoire de Tours, la documentation remonte jusqu'au quinzième siècle. Le chapitre III contient une masse de considérations diverses. Une première partie fait

21 B. Belhoste « Un modèle à l'épreuve. L'École Polytechnique de 1794 au second Empire ॥, op. cit. ; Olivier Darrigol, " Between hydrodynamics and elasticity theory: the five first births of the Navier-Stokes equations "), Archives for the History of Exact Sciences 56, 2002, pp. 127-130, en particulier, pp. 95-150.

22 Ces écrits ont été analysés par K. Chatzis, « Les conceptions de Barré de Saint Venant (1797-1886) en matière de théorie de la connaissance $॥$, op. cit. On retrouve aussi cette position dans de nombreux endroits de sa correspondance avec Boussinesq conservée à la bibliothèque de l'Institut de France (MS4226 et MS4227). l'état des lieux avant les travaux. La suivante s'intitule « Choix à faire entre les différents modes d'alimentation de Dijon ॥. Ses dix pages sont en bonne partie consacrées à l'étude des formules d'où on peut déduire le volume d'eau nécessaire à l'alimentation d'une ville, avec de nombreux exemples. La troisième partie de ce chapitre est un peu bizarrement intitulée "D'après le résultat trouvé pour le volume d'eau nécessaire à l'alimentation d'une ville ॥. Darcy y justifie en détail le choix de la source du Rosoir. Suivent encore cinq parties intitulées:

- De l'origine des fontaines

- Singularités que les fontaines offrent quelquefois à l'observateur

- Des moyens de découvrir et de créer des sources

- Des puits artésiens

- Application des principes développés dans les pages ci-dessus relatées [à un certain nombre de sources situées en différents endroits de France]

La table des matières de ce chapitre indique in fine: «Voir (note D) les conclusions que permet de tirer la loi expérimentalement démontrée de l'écoulement de l'eau à travers les sables, en ce qui concerne:

$1^{\circ}$ Le décroissement du débit des sources à partir de leur étale;

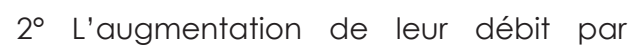
l'abaissement artificiel de leur niveau ॥.

La deuxième partie est consacrée à une description très détaillée du système d'adduction d'eau de Dijon, y compris les procédés d'exécution et les coûts. La troisième, appelée «Expériences » décrit de nombreuses mesures faites sur ce système en $y$ ajoutant des réflexions plus générales, en particulier sur les formules qui donnent le débit des tuyaux en fonction de la différence de pression que l'eau y subit et de leur diamètre. Ces expériences ont eu lieu en 1846 et 1853, les unes avant et les autres après celles qui sont rapportées dans le livre de 1857 sur le mouvement de l'eau dans les tuyaux. Enfin la quatrième partie traite des questions administratives (expropriations etc).

\section{La loi de Darcy in statu nascendi}

La fin du livre sur les fontaines de la ville de Dijon est un appendice constitué de nombreuses notes. Une partie de la note D intitulée : " Détermination des lois d'écoulement de l'eau à travers du sable » 


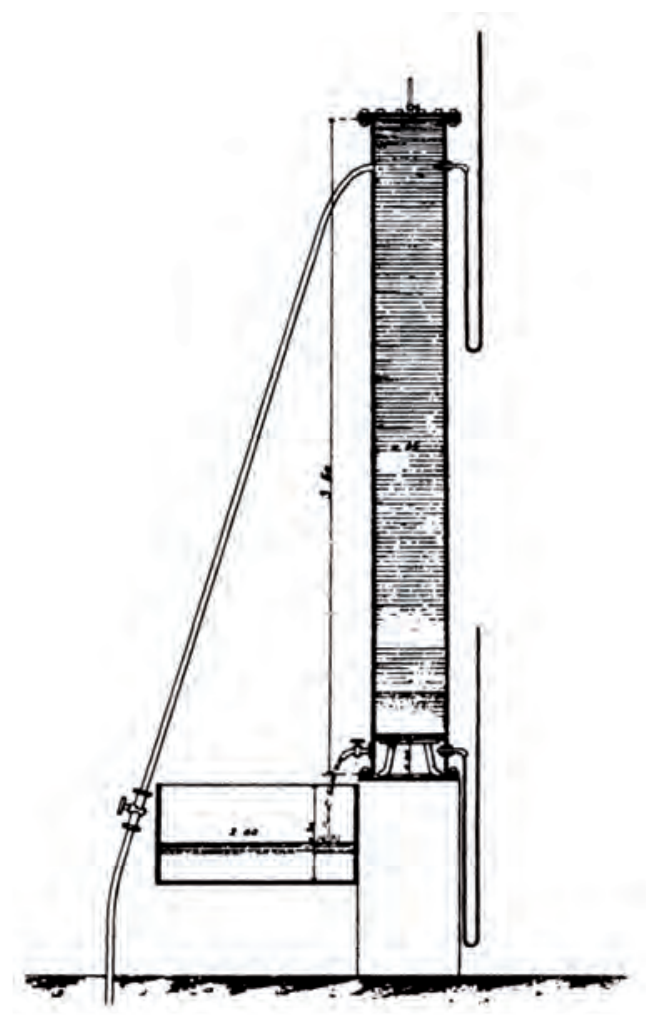

fig. 1 - Le dispositif de vérification de la loi de Darcy (extrait de Darcy Les fontaines publiques de la ville de Dijon, planche 24)

se trouve dans les pages 590 à 594. Darcy y vérifie expérimentalement que :

$$
q=k s / e(h+e)
$$

où q est le débit, s la surface de la section de la couche de sable, e son épaisseur et $h$ la différence de pression entre le sommet et la base de la couche exprimée en hauteur d'eau; enfin $k$ est un coefficient qui dépend de la nature du sable utilisé. Le facteur $h+e$ est la charge hydraulique ${ }^{23}$.

La forme moderne est locale et différentielle :

$$
v=-(K / \mu) \operatorname{grad}(p+V)
$$

où $v$ est la vitesse, $K$ un coefficient qui ne dépend que du milieu poreux, $\mu$ la viscosité dynamique ${ }^{24}, p$

23 La charge hydraulique est la somme de la pression et du potentiel des forces extérieures qui s'exercent sur un fluide. La perte de charge entre deux points est la différence entre la charge hydraulique au point le plus en amont et l'autre. C'est donc aussi la force motrice par unité de surface qui s'exerce sur le fluide compris entre ces deux points. 24 La viscosité d'un fluide est un paramètre qui caractérise l'interaction entre deux filets fluides contigus. Selon la situation étudiée, on utilise la viscosité cinématique ou la pression et $V$ le potentiel d'un éventuel champ de forces extérieures, en général la pesanteur ${ }^{25}$. II faut parfois ajouter une correction de capillarité. Pourvu que les vitesses ne soient pas trop grandes, elle est valable pour tout écoulement en milieu poreux, même s'il n'est pas stationnaire ${ }^{26}$.

L'appareil employé pour les expériences (voir la figure 1, qui est extraite de la planche 24) était un cylindre vertical de $0,35 \mathrm{~m}$ de diamètre intérieur et de 3,5 m de hauteur fermé par une plaque aux deux bouts. À 0,2 m au-dessus de la base, on a placé un système de grilles qui supporte la couche de sable. Chacune des deux parties non occupées par le sable est munie d'un robinet à eau, d'un robinet à air qui permet de faire varier la pression tant dans celle du bas que dans celle du haut (sur la figure, on ne voit pas les robinets à air) et d'un manomètre ${ }^{27}$ à mercure. Le robinet à eau du bas coulait dans un bassin permettant de jauger le volume d'eau écoulé. La couche filtrante est formée d'un mélange de sables de la Saône de finesses diverses dont $58 \%$ passant au crible de $0,77 \mathrm{~mm}$ (c'est le plus fin). $38 \%$ du volume est laissé vide par le sable.

Avant chaque série d'expériences, on mettait dans la colonne remplie d'eau une certaine quantité de sable. Chaque expérience consistait à établir une différence de pression entre le haut et le bas de la colonne de sable, à attendre que le débit se stabilise et à mesurer le volume écoulé dans le bassin de jauge pendant un certain temps (10 à 20 minutes selon les expériences). La pression présentait quelques oscillations pendant chaque expérience. En effet, l'appareil était installé dans un hôpital et le contrecoup de l'ouverture et de la fermeture d'un autre robinet dans l'établissement pouvait se faire sentir. Mais ces oscillations ne dépassaient pas $1 \%$ de la différence de pression.

La figure 2 (page suivante) donne le résultat de ces expériences tel qu'on le trouve dans le livre de

la viscosité dynamique, produit de la précédente par la masse volumique. La viscosité dépend de la température. 25 grad désigne traditionnellement le gradient, c'est-à-dire le vecteur ayant pour composantes les dérivées partielles.

26 Rappelons qu'un écoulement est dit stationnaire lorsque les vitesses ne dépendent pas du temps. S'il s'agit de l'écoulement d'un liquide (fluide incompressible) dans un tuyau, il faut alors que la vitesse moyenne reste la même tout le long du tuyau. La force qui meut l'eau renfermée entre deux sections du tuyau est alors le produit de la perte de charge par la surface de la section. Puisque l'écoulement est stationnaire, elle est égale à la force qui s'oppose à l'écoulement de l'eau.

27 Darcy emploie indifféremment les mots « manomètre » et " piézomètre " pour les baromètres. 


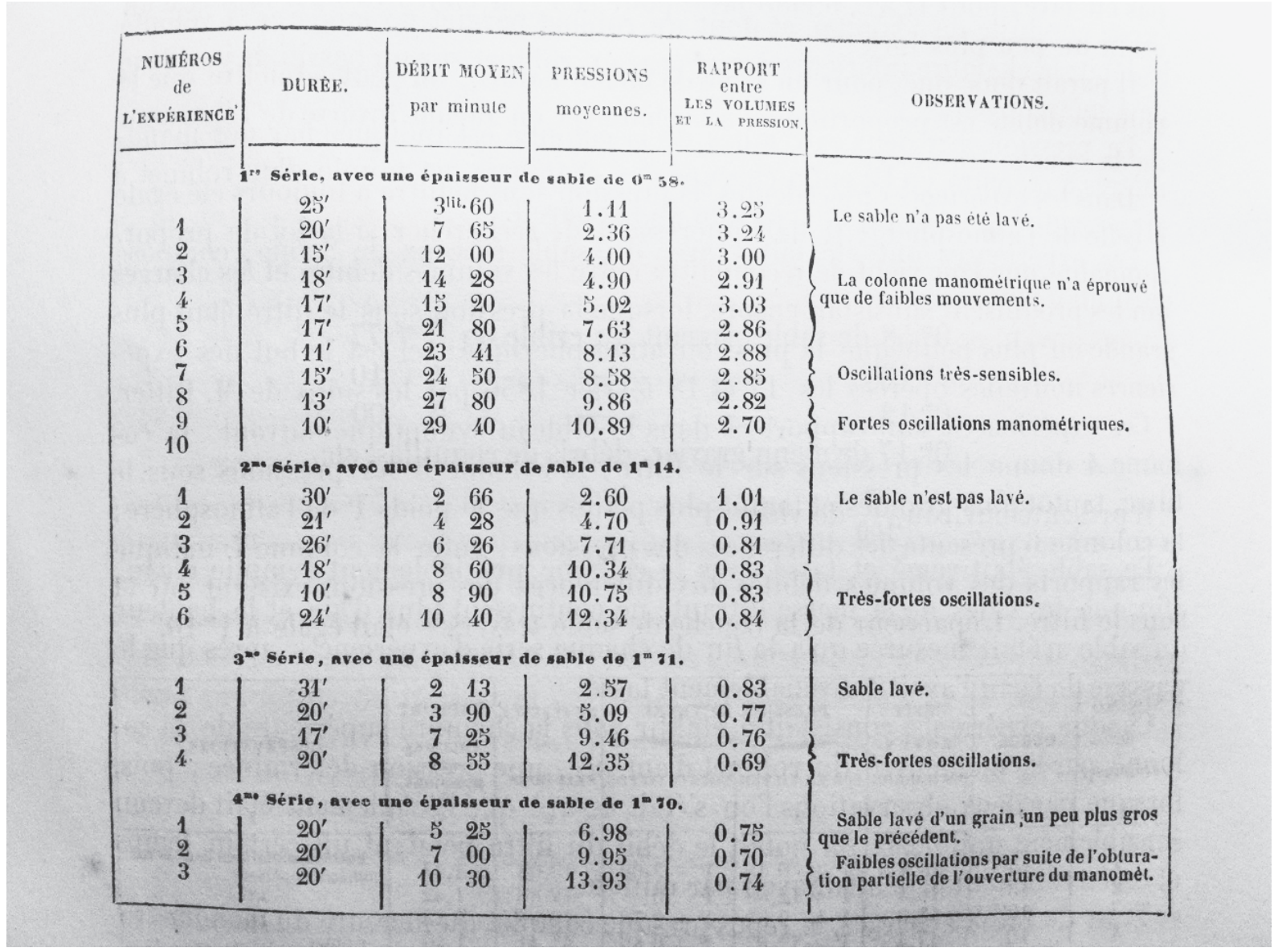

fig. 2 - Tableau des expériences faites à Dijon les 29 et 30 octobre et 2 novembre 1855 (extrait de Darcy, Les fontaines publiques de la ville de Dijon, p. 592).

(C) Bibliothèque centrale du Conservatoire National des Arts et Métiers, Paris

Darcy (page 592). Les pressionssontexprimées enmètres d'eau. «Toutes les pressions ont été rapportées à la base du filtre ॥, ce qui doit signifier que l'on a ajouté l'épaisseur de la colonne de sable (le terme e de la formule) et qu'il s'agit donc de charges plutôt que de pressions.

Citons Darcy : « le débit par seconde et par mètre carré est lié très-approximativement à la charge par les relations suivantes $\|$ :

$$
\begin{array}{cccc}
1^{\text {ère }} \text { série } & 2^{\text {ème }} \text { série } & 3^{\text {ème }} \text { série } & \text { 4ème série } \\
Q=0,493 P & Q=0,145 P & Q=0,126 P & Q=0,123 P
\end{array}
$$

Mis à part le remplacement du litre par minute par le litre par seconde, il s'est livré aux opérations suivantes. II a calculé dans chaque série un coefficient qui est le rapport de la moyenne des pressions à la moyenne des débits divisée par la section de la colonne $\left(0,096 \mathrm{~m}^{2}\right)$. Le facteur s de la formule n'est pas expérimental, Darcy sait d'avance que le débit est proportionnel à la surface de la section. En prenant directement la moyenne des rapports des débits aux pressions (colonne 5), on s'aperçoit que les différences atteignent environ $10 \%$ de part et d'autre (elles ne peuvent donc pas s'expliquer par les incertitudes sur la mesure de la pression). Ces différences n'inquiètent pas Darcy. Par contre, la pression sous la colonne de sable étant toujours la pression atmosphérique dans ces premières expériences, il prendra la précaution d'en faire faire une autre où elle varie. Cette nouvelle série d'expériences n'appelle pas de commentaire particulier.

II reste à étudier l'influence de l'épaisseur e de la couche de sable. Pour cela, Darcy appelle I la charge proportionnelle par mètre d'épaisseur du filtre, c'est-à-dire $P / e$ et il trouve:

$$
\begin{array}{cccc}
1^{\text {ère }} \text { série } & 2^{\text {ème }} \text { série } & 3^{\text {ème }} \text { série } & 4^{\text {ème }} \text { série } \\
Q=0,286 \mid & Q=0,165 \mid & Q=0,2161 & Q=0,3321
\end{array}
$$

Ces résultats sont faciles à contrôler et on s'aperçoit par la même occasion que l'épaisseur de 1,70 $\mathrm{m}$ qu'on trouve sur le tableau pour la quatrième série d'expériences est une erreur, il s'agit de 2,70 m (on trouve d'ailleurs d'autres coquilles dans les données numériques du livre). 
Ces rapports devraient être tous les mêmes et on voit qu'ils varient du simple au double. Darcy l'explique par des différences dans la nature et le traitement des sables employés et conclut: " Il paraît donc que, pour un sable de même nature, on peut admettre que le volume débité est proportionnel à la charge et en raison inverse de l'épaisseur de la couche traversée ".

Cette loi ainsi établie, Darcy en déduit celle qui donne en fonction du temps la hauteur d'une nappe d'eau qui s'écoule à travers une couche de sable. Il s'agit d'écrire et de résoudre une équation différentielle. Le point clef est évidemment que la dérivée par rapport au temps de la hauteur d'eau est égale au signe près au débit divisé par la surface. Ce passage est à considérer comme banal compte tenu de la culture scientifique de l'époque. Enfin, en utilisant de nouveau l'appareil déjà décrit, il vérifie expérimentalement la relation ainsi trouvée, confirmant par là la loi de Darcy ellemême. L'accord avec l'expérience est d'ailleurs plus satisfaisant (environ 5\%).

De ce qui précède il serait naïf de conclure que Darcy a trouvé sa loi en ajustant, grossièrement au demeurant, les mesures par une fonction linéaire. II y a toutes les raisons de penser qu'il vérifiait expérimentalement une conclusion déjà tirée ${ }^{28}$. Nous allons nous efforcer d'expliquer comment il y est parvenu à partir de son étude antérieure de deux phénomènes: les puits artésiens et l'écoulement de l'eau dans les tuyaux. Pour éclaircir le rapport que Darcy a pu leur trouver avec sa loi, il faut commencer par essayer de mieux préciser la chronologie de ses travaux.

\section{Retour sur la chronologie des travaux de Darcy}

Nous avons dit que le livre sur les fontaines de Dijon était un véritable monde. De nombreux éléments devaient déjà se trouver dans le rapport de 183429, mais ce ne sont pas ceux qui nous concernent directement.

Il est difficile de dater le début de l'intérêt de Darcy pour les puits artésiens. Le sujet était d'actualité dans le corps des Ponts et Chaussées depuis les années 1820 au moins ${ }^{30}$. A la même

28 C'est aussi l'avis de Simmons, Henry Darcy (1803-1858), op. cit., avec une argumentation différente de la mienne.

29 Pour une description succincte de ce rapport : Lochot "L'homme et ses réalisations », op. cit. pp. 10-12).

30 De 1820 à 1840, le nombre des puits artésiens se multiplie en France : 12 à Paris, 8 à Saint-Denis, 3 à Mulhouse. D'autres sont creusés avec succès au Havre, à Tours, Strasbourg, La Rochelle, Perpignan. Voir Jean-Pierre Goubert, La conquête époque, Darcy mesure le débit d'un puits artésien qui se trouvait à Dijon pour conclure qu'il ne pouvait pas suffire à l'alimentation en eau de la ville. Dans le livre sur les fontaines de Dijon, il rapporte les résultats d'expériences faites sur les puits de Tours, sur le célèbre puits artésien de Grenelle à Paris et enfin sur le puits de Villainesen-Duesmois. Ce dernier se trouve dans la circonscription de Dijon. II a été percé en 1847 et les expériences ont été faites par Darcy lui-même la même année, les mesures sur les autres puits sont antérieures ${ }^{31}$. Nous pouvons donc au moins être sûrs de son intérêt actif pour la question sans savoir à partir de quel moment il a entrepris une étude systématique, probablement bien avant les expériences sur les conduites. Cette partie du livre contient une loi liant le débit d'un puits artésien à la hauteur de son ouverture ${ }^{32}$.

Les dernières expériences rapportées dans la note $D$ ont été faites par l'ingénieur des Ponts et Chaussées Ritter le 18 février 1856. II n'y a pas d'achevé d'imprimer, mais nous savons que le livre est paru la même année. Cela nous amène à penser que cette note a été écrite rapidement, opinion confortée par plusieurs coquilles, alors qu'en général l'écriture est très soignée. La hâte est confirmée par la mention tout à fait inusuelle de la note $D$ dans la table des matières juste après le chapitre III de la première partie.

Lorsque Darcy a été chargé de l'étude de l'adduction d'eau de Dijon, le corps des Ponts et Chaussées pouvait s'appuyer sur une tradition d'étude du mouvement de l'eau dans les conduites qui remontait à du Buat (1734-1809). On savait que, dans un écoulement stationnaire de section constante, la perte de charge est égale à la force retardatrice divisée par la section, ainsi qu'il résulte d'un bilan de quantité de mouvement ${ }^{33}$. Les opinions

de l'eau, Paris, Hachette 1990, p. 55. Sur les forages artésiens à Paris sous la Restauration, dont le puits de Grenelle, Philippe Cebron de Lisle, L'eau à Paris au XIXe siècle, Paris, AGHTM, 1991, p. 187-189. Sur la théorie des puits artésiens au XVIII siècle et aux premières décennies du siècle suivant : André Guillerme, Les temps de l'eau. La cité, l'eau et les techniques, Seyssel, Editions du Champ Vallon, 1983, pp. 193-194.

31 H. Darcy, Les fontaines, op. cit. pp. 175-178).

32 lbid., pp. 156-157.

33 La quantité de mouvement, ou impulsion, d'un point matériel est le produit de sa vitesse et de sa masse. La loi de Newton peut donc s'exprimer en disant que la dérivée de la quantité de mouvement par rapport au temps est égale à la force. La quantité de mouvement d'un système est la somme des quantités de mouvement de ses composants ou leur intégrale selon qu'il s'agit d'un système discret ou continu. 
divergeaient sur la nature de cette force retardatrice mais l'accord se faisait pour la dire proportionnelle à $a u+b u^{2}, u$ étant la vitesse moyenne et $a$ et $b$ des coefficients à déterminer expérimentalement. On utilisait pour la perte de charge dans une conduite d'eau cylindrique la formule due à Gaspard Riche de Prony (1755-1839) :

$$
2 L\left(0,0000188 u+0,000343 u^{2}\right) / R
$$

où $L$ est la longueur de la conduite, $u$ la vitesse moyenne, $R$ le rayon, les unités le mètre et la seconde ${ }^{34}$.

Prony avait obtenu ces valeurs des coefficients par ajustement aux résultats des trois séries d'expériences faites par ses prédécesseurs, le premier étant du Buat, pour disposer de plus de mesures. Le défaut était qu'il s'agissait de mesures faites sur des tuyaux de natures très différentes, les uns neufs, d'autres déjà encrassés. Aussi ne faut-il pas s'étonner que la formule ait été parfois trouvée en défau ${ }^{35}$. $\|$ est difficile de savoir quand Darcy a commencé à avoir des doutes à ce sujet, au plus tard en 1849 puisque ses expériences parisiennes se sont déroulées du 31 août 1849 au 27 octobre 1851 . Probablement s'est-il posé la question plus tôt puisqu'il écrit : " J'ai donc voulu reconnaître si le débit de ces derniers [łuyaux] avait diminué d'une valeur sensible au bout de sept années $11^{36}$. D'autres mesures avaient été faites à Dijon en $1846^{37}$. Cette formule avait déjà causé des déboires aux ingénieurs des Ponts et Chaussées

Les Recherches expérimentales relatives au Mouvement de l'Eau dans les Tuyaux sont parues en 1857 mais ont été écrites beaucoup plus tôt sous forme d'un mémoire à l'Académie des Sciences. La formule de Prony était la doctrine des Ponts et Chaussées et l'autorité de Prony était grande. Darcy tenait donc à assurer ses arières et a demandé à l'Académie un rapport avant de publier l'ouvrage. Ce rapport, écrit par le polytechnicien Arthur Morin (1795-1880)38 figure aux Comptes Rendus à la date du 26 juin 1854. II est reproduit au début du livre de 1857. C'est dire que le mémoire a été écrit entre fin 1851 et début 1854, en tout cas avant le livre sur les fontaines qui le mentionne à plusieurs reprises et cite le rapport de Morin ${ }^{39}$.

34 Sur cette question : Hippolyte Sonnet, Dictionnaire des Mathématiques appliquées, Paris, Hachette, 1867, article Conduites, pp. 288-291.

35 Voir 0 . Darrigol Worlds of flows, op. cit. qui étudie le mémoire de Darcy de ce point de vue.

$36 \mathrm{H}$. Darcy Les fontaines, op. cit., p. 419.

37 Ibid., pp. 420-421.

38 Sur Morin, voir la contribution de Claudine Fontanon dans ce volume.

39 Les deux autres commissaires sont Poncelet et Combes.

\section{Le dispositif expérimental de Paris}

Nommé directeur du service des eaux et pavés de la ville de Paris, Darcy disposait de moyens considérables pour étendre et préciser les mesures faites à Dijon. II choisit d'utiliser les installations de la colline de Chaillot qui fournissaient une bonne partie de l'eau consommée à Paris. La figure 3 aidera à comprendre la description du dispositif40. II s'agit d'une batterie de pompes alimentant une cuve située à trois ou quatre cents mètres de la Seine et 47,2 m au-dessus de son niveau à l'étiage à travers un tuyau de $25 \mathrm{~cm}$ de diamètre. Elle alimentait aussi, à travers un tuyau de $65 \mathrm{~cm}$, quatre bassins situés une quinzaine de mètres plus bas.

Pour réaliser ses expériences, Darcy fit faire des appareils et réaliser des travaux. Trois tuyaux verticaux de diamètres différents constituaient des prises d'eau sur la partie inférieure de la conduite de $65 \mathrm{~cm}$ et un sur celle de $25 \mathrm{~cm}$. Ils étaient tous munis de robinets débouchant dans une conduite de 30 $\mathrm{cm}$. Cette conduite d'un peu plus de $20 \mathrm{~m}$ de long aboutissait dans un cylindre horizontal fermé. De ce cylindre partait la conduite sous expérience qui aboutissait à son tour dans un autre cylindre, celuici vertical et ouvert, qui se déchargeait dans un bassin de jaugeage. Cette conduite était presque horizontale mais avec une légère pente vers l'amont pour faciliter l'évacuation des bulles d'air. Le cylindre situé à l'extrémité amont de la conduite était muni d'un dispositif permettant de lui adapter des tuyaux de diamètres différents. Le réglage de la charge hydraulique se faisait en ouvrant plus ou moins un ou deux des robinets de dérivation et aussi en fixant plus ou moins haut le niveau du plus bas des quatre bassins situés sur la colline. Un appareil particulier permettait ce réglage pour les deux conduites les plus étroites, celles qui nous intéressent ici ${ }^{41}$.

Cinq manomètres étaient installés sur le trajet. Darcy les numérote d'aval en amont. Le cinquième était placé sur le cylindre amont près de la sortie et le quatrième sur la conduite en expérience près de l'entrée. La donnée utilisée dans l'interprétation des expériences était la perte de charge entre le troisième manomètre, placé à 4,7 m après celui de l'entrée et le premier situé $100 \mathrm{~m}$ plus loin, sauf dans quelques expériences qui ne nous concernent pas. Un manomètre de contrôle était placé à mi-chemin entre le premier et le troisième. La charge la plus élevée (celle du

40 Extraite de : Henry Darcy, Recherches expérimentales relatives au Mouvement de l'Eau dans les Tuyaux, Paris, Mallet-Bachelier, 1857, planche 2.

41 H. Darcy Recherches expérimentales ... op. cit., pp. 31-32 


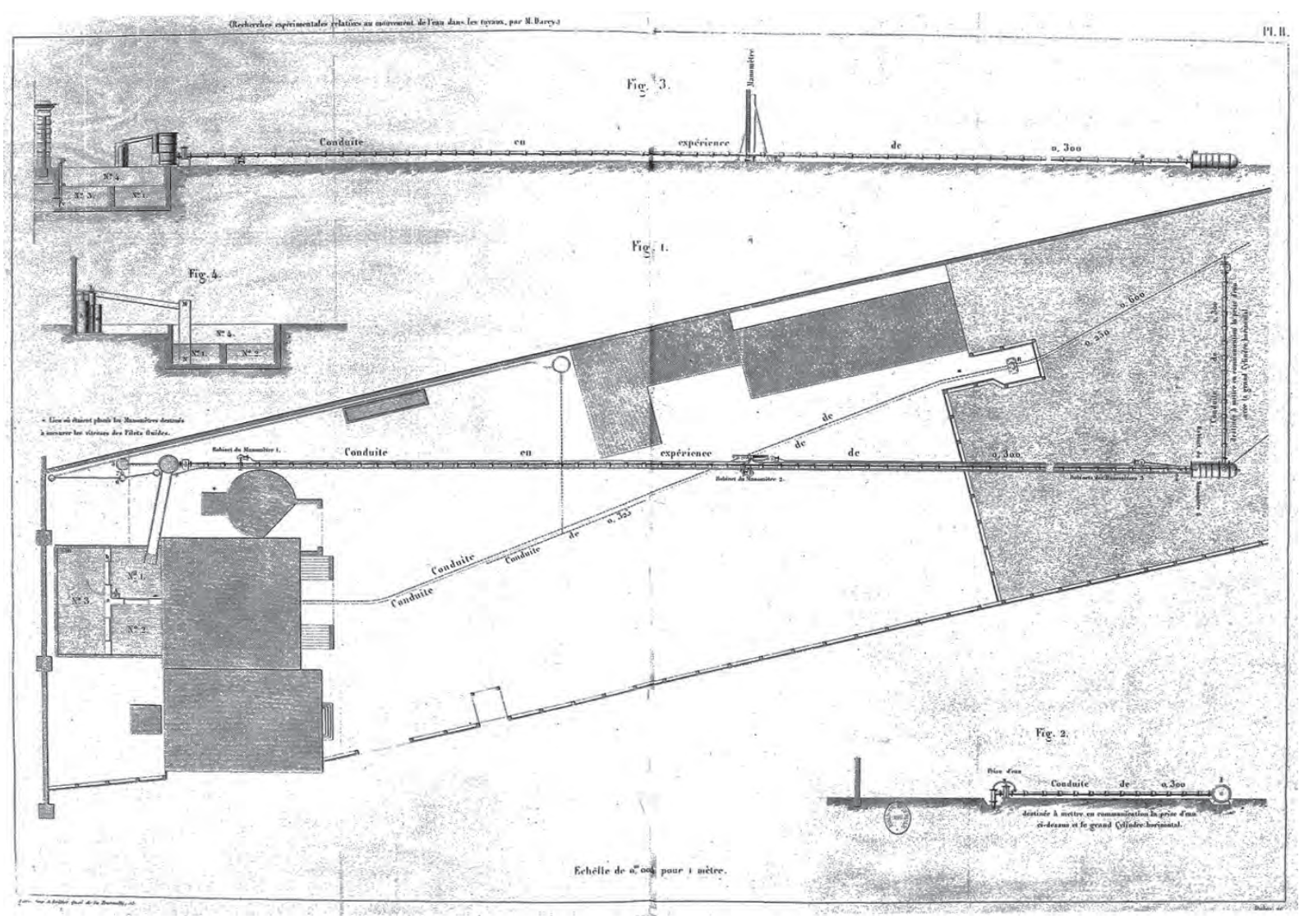

cinquième manomètre) varie depuis moins de un mètre à plus de vingt selon les expériences et les vitesses de 0,03 jusqu'à 5 ou $6 \mathrm{~m} / \mathrm{s}^{42}$.

On conçoit que la mise en place de ce dispositif a nécessité des travaux importants. Les expériences ont été faites sur vingt-deux conduites de diamètres différents et de matériaux divers. Celles qui nous intéressent sont les deux plus minces d'entre elles, de 0,0122 et $0,0266 \mathrm{~m}$ de diamètre, toutes deux en fer étiré. Chaque changement de conduite exigeait le calibrage et la pose de $100 \mathrm{~m}$ de tuyaux, parfois un changement dans le dispositif. A chaque nouvelle expérience, il fallait changer le réglage de la charge, puis attendre que l'écoulement prenne son régime stationnaire. Darcy donne la durée de chaque expérience, mais sans préciser ce qui était considéré comme le début et la fin. Dans un cas, il a fallu un jour et une nuit rien que pour purger les manomètres ${ }^{43}$.

\section{Les écoulements lents}

Le but principal des expériences de Darcy sur les conduites est de fournir aux ingénieurs chargés des adductions d'eau un moyen fiable de calculer le débit en fonction de la perte de

42 lbid. pp. 46 sqq. les tableaux donnant les résultats des 198 expériences.

43 Ibid., p.34. fig. 3 - Plan de l'installation de Chaillot (partie basse) avec le dispositif expérimental de Darcy (extrait de Darcy Recherches expérimentales relatives au Mouvement de l'Eau dans les Tuyaux, planche 2)

charge hydraulique. Presque toutes ses mesures portent donc sur des situations qu'ils rencontrent usuellement. Cependant, il en profite pour étudier deux autres questions. L'une est la répartition des vitesses à l'intérieur des tuyaux, l'autre, celle qui nous intéresse, la formule à utiliser pour les faibles vitesses ${ }^{44}$.

Résumons la réponse dans les termes de Morin : " L'auteur fait remarquer, en outre, que, pour les petites vitesses inférieures à $0^{m}, 10$ par seconde, le terme relatif au carré de la vitesse dans les formules de résistance paraît avoir si peu d'influence, que cette résistance devient proportionnelle à la simple vitesse $11^{45}$.

44 La question des faibles vitesses (entre autres, le comportement de l'eau dans le sol) est une question ancienne qui a intéressé depuis la fin du XVIII ${ }^{e}$ siècle plusieurs ingénieurs du corps des ponts et chaussées et du corps du Génie, dont Coulomb, Prony et Girard. Voir les analyses contenues dans : André Guillerme, Bâtir la ville. Révolutions industrielles dans les matériaux de construction, France-Grande-Bretagne (17601840), Seyssel, Champ Vallon, 1995, p. 99, p. 106, p. 144 et passim). 45 Cité d'après H. Darcy, Recherches expérimentales, op. cit., p. xil. 
Il faut comprendre que la formule de Prony est à remplacer par : $H / L=a u$ où $H$ est la perte de charge et le coefficient a dépend du tuyau considéré.

Pour vérifier cette nouvelle formule, il faut disposer d'au moins trois mesures, deux à la rigueur (la droite qui joint les deux points expérimentaux doit passer par l'origine). Or les vitesses sont très rarement inférieures à $0,1 \mathrm{~m} / \mathrm{s}$ dans les conduites. Sur les vingtdeux conduites testées par Darcy, seules les trois faites de fer étiré permettent cette vérification. Elles portent les numéros 1 à 3 respectivement, les tuyaux sont en fer étiré et les diamètres des deux plus minces valent $0,0122 \mathrm{~m}$ et $0,0266 \mathrm{~m}$. On peut remarquer que la vitesse de $10 \mathrm{~cm} / \mathrm{s}$ correspond à des nombres de Reynolds ${ }^{46}$ de 1000 et 2000 respectivement pour les deux premières, à comparer à 2000, la limite au-delà de laquelle on ne peut pas espérer trouver un écoulement de Poiseuille. En fait, Darcy trouve au départ trois mesures utilisables pour le tuyau $n^{\circ} 1$, deux pour le $n^{\circ} 3$ et une seule pour le $n^{\circ} 2$. II décide alors de faire quatre mesures complémentaires sur ce dernier. Le choix de ce tuyau plus large est difficile à comprendre, d'autant plus que : "La surface du tuyau de 0m,0266 était beaucoup plus rugueuse que celle des deux autres. II a pu en résulter des erreurs dans la mesure du diamètre $\|^{47}$.

Des mesures complémentaires sur la conduite $n^{\circ} 1$ auraient pu lui montrer que la limite en deçà de laquelle la formule en $u$ est valable dépendait de la largeur du tuyau considéré. Mais on ne refait pas I'histoire. Darcy remarque :

"On sait que dans les tuyaux capillaires, M. Poiseuille a trouvé la formule :

$$
\Phi=K D^{4} \mathrm{H} / \mathrm{L}
$$

la même que ci-dessus; résultat assez remarquable, puisque nous sommes parvenus, $M$. Poiseville et moi, à cette expression, au moyen d'expériences faites dans des circonstances tout à fait différentes. L'expression générale précédente paraît donc renfermer le lien qui unit les lois de l'écoulement dans un tuyau de diamètre quelconque et dans un tuyau capillaire $11^{48}$.

46 Le nombre de Reynolds d'un écoulement est le produit d'une longueur et d'une vitesse typiques divisé par la viscosité cinématique. Il est sans dimension (il ne dépend pas des unités choisies). Les écoulements réguliers deviennent instables au delà d'un certain nombre de Reynolds, environ 2000 pour les écoulements de Poiseville.

47 H. Darcy, Recherches expérimentales, op. cit., p. 213. 48 H. Darcy Recherches expérimentales, op. cit., p. 213, suite $\phi$ est le débit en volume. La citation se retrouve presque mot pour mot dans le livre sur les fontaines, (note H, p. 639).

Maintenant, nous savons calculer K. Nous pouvons donc, à partir des tableaux donnés par Darcy, calculer les vitesses moyennes des écoulements de Poiseville correspondants. Les valeurs expérimentales se trouvent légèrement en dessous avec des écarts allant de $5 \%$ à $22 \%$. Parmi les causes possibles des écarts, les ajutages des tuyaux, la rugosité du tuyau $n^{\circ} 2$, des incertitudes sur la viscosité, Darcy ne donnant pas toujours la température de l'eau.

\section{Quelle interprétation ?}

La citation de Morin ci-dessus peut laisser penser que la loi linéaire trouvée par Darcy pour les petites vitesses tenait simplement au fait que dans la formule de Prony, ou mieux dans les formules de même forme trouvées pour les différents tuyaux, le terme en $u^{2}$ devient négligeable devant celui en $u$. II n'en est rien. Avec une vitesse de $10 \mathrm{~cm} / \mathrm{s}$, le premier est encore le double du second dans la formule de Prony. Le rapport est plus petit avec les coefficients trouvés par Darcy pour les tuyaux concernés ${ }^{49}$, mais au moins proche de un. Ces évaluations ne se trouvent ni chez Morin ni chez Darcy. Cependant ce dernier s'est aperçu d'une autre manière, plus graphique et visuelle, qu'il s'agissait d'autre chose:

"Lorsque l'on jette les yeux sur le tracé des courbes [qui donnent la vitesse en fonction de la pente] dont le tableau précédent renferme les éléments, on s' aperçoit que dans les environs de l'origine des coordonnées, près de la vitesse $0^{m}, 08$ ou 0m,09, ces courbes ne semblentpas assujetties à cetteloi de continuité qui préside ordinairement à la succession des phénomènes naturels découlant d'une loi unique (planche XII). A partir de l'extrémité de la ligne droite qui passe par l'origine, extrémité qui se rencontre aux abords de $0^{m}, 10$, la ligne s'infléchit assez brusquement pour suivre une courbure parabolique, de telle sorte que la droite semble inscrite à cette dernière. II semblerait donc qu'en franchissant la vitesse de $0^{m}, 10$ un fait nouveau se produise, lequel vient porter atteinte à la loi de continuité $1^{50}$.

d'une note commencée à la page précédente.

49 Les résultats se compliquent parce que, toujours très consciencieux, Darcy utilise deux méthodes d'ajustement différentes.

50 H. Darcy Recherches expérimentales, op. cit., p. 214. ॥ y revient à la page suivante : «Quoi qu'il en soit, il semble que deux lois apparaissent dans le phénomène produit par 
Le « fait nouveau » avait été mis en évidence dès 1839 par Hagen ${ }^{51}$. Ni Darcy ni Morin ne le mentionnent et nous pouvons être assurés qu'ils ne le savaient pas. Notons que Hagen faisait en laboratoire des expériences spécialement conçues pour l'étude des écoulements de faible vitesse, tout le contraire de Darcy qui ne consacre que très peu de ses mesures à ce phénomène. II semble aussi que Hagen, et pas Darcy, voyait l'eau de ses expériences s'écouler et pouvait donc constater que le débit devenait irrégulier. On trouve ici une limite des expériences grandeur nature mal adaptées à l'étude d'écoulements lents. Quoi qu'il en soit, en 1856 et sans doute dès 1851, Darcy considère comme acquise la proportionnalité de la vitesse à la perte de charge dans les tuyaux aux petites vitesses.

\section{Les puits artésiens}

Le chapitre III de la première partie des Fontaines publiques de la Ville de Dijon consacre une quarantaine de pages (à partir de la page 137) aux puits artésiens. II rapporte en particulier des résultats de mesures de débit en fonction de la hauteur à laquelle l'eau se déverse. Et il ajoute : « Je terminerai ce que j'avais à dire sur les puits artésiens par quelques considérations relatives à la courbe que l'on obtient en réunissant par une ligne les extrémités des perpendiculaires élevées sur le tube ascensionnel aux différentes hauteurs de déversement des eaux; ces perpendiculaires renfermant autant d'unités linéaires que le volume correspondant comprend lui-même d'unités. Si l'on jette les yeux sur la planche 22, où sont tracées quelques unes de ces courbes, on verra qu'en général elles diffèrent très-peu d'une ligne droite. Pourquoi ? On lira au chapitre ॥ de la troisième partie de cet ouvrage que, dans les vitesses comprises entre zéro et dix ou onze centimètres par seconde, l'expérience a toujours montré que les vitesses ou les volumes étaient proportionnels aux pentes.

Or dans les masses sablonneuses, les eaux doivent circuleren général avec des vitesses encore inférieures à dix ou onze centimètres, et par conséquent il est probable qu'elles cheminent suivant la loi précitée(').

(1) Cette supposition conduisant à la loi généralement trouvée, comme on va le voir, pour les puits artésiens, on pourrait réciproquement conclure de l'existence expérimentale de cette loi que la supposition initiale est fondée $\|^{52}$.

l'écoulement de l'eau dans les tuyaux de conduite et que ces lois, au moins dans les diamètres que j'ai employés, viennent se souder vers les vitesses de 9 à 10 centimètres $॥$.

51 Gotthilf Hagen, Uber die Bewegung des Wassers in engen zylindrischen Röhren Annalen der Physik 461839 ; O. Darrigol, Worlds of flows, op. cit. pp. 140-14.

$52 \mathrm{H}$. Darcy, Les fontaines, p. 156 et note en bas de page.
II faut comprendre que le tube ascensionnel (vertical) qui part de la nappe aquifère est percé de divers orifices et qu'on mesure les débits en en laissant ouvert un seul. Pour les courbes de la planche 22, ce tube estl'axe (horizontal) des abscisses. Les ordonnées sont les débits. Ces courbes sont reproduites dans la figure 4. Au passage, notons une fois de plus la nature graphique du raisonnement. Darcy ne décrit pas en détail le dispositif expérimental qui a d'ailleurs pu varier d'un puits à l'autre.

La constatation expérimentale est donc que le débit $q$ est proportionnel à la distance $h$ de l'orifice au point le plus haut atteint par l'eau. Cette distance est égale à la perte de charge (exprimée en hauteur d'eau évidemment).

On peut en conclure que ce passage a été écrit avant les expériences établissant la loi de Darcy. D'un autre côté il ne peut pas être antérieur aux expériences sur les conduites. On aimerait savoir quand Darcy a constaté la "loi des puits artésiens". Dans ce passage, c'est une conséquence de celle qui régit les écoulements lents, étendue implicitement des tuyaux aux sables ${ }^{53}$. Dans le chapitre consacré aux mesures de débits faites à Dijon, Darcy écrit : "Les formules relatives au mouvement de l'eau dans les tuyaux de conduite sont, comme on vient de le voir, $R i=a v+b v^{2}$ ou $R i=b_{1} v^{2}$; ce n'est que dans des cas très particuliers que l'on peut adopter la relation $\mathrm{Ri}=\mathrm{a}_{1} \mathrm{v}^{(1)}$.

(1) On verra dans mon mémoire surl'écoulement de l'eau dans les tuyaux de conduite, que cette relation a été vérifiée par l'expérience dans les tuyaux de petit diamètre et où la vitesse du fluide ne dépassait pas dix à douze centimètres par seconde. J'ai démontré, par des expériences spéciales, (voir la note $D$ relative au filtrage), que, dans l'écoulement de l'eau à travers le sable fin, le débit était proportionnel à la charge. Ainsi se trouve justifié l'aspect théorique de la page 156, en ce qui concerne les puits artésiens alimentés par des couches sablonneuses aquifères $\|^{54}$.

Ri est la force qui s'oppose à l'écoulement de l'eau dont la nature n'était pas encore éclaircie, $v$ est la vitesse de l'eau et les $a$ et $b$ des coefficients à déterminer expérimentalement. On remarque que la référence à la loi de Darcy se trouve dans la note qui a pu être ajoutée tardivement.

53 Pourvu qu'on puisse négliger les pertes de charge dans le tube ascensionnel.

$54 \mathrm{H}$. Darcy, Les fontaines, op. cit. p. 384 et note en bas de page. 


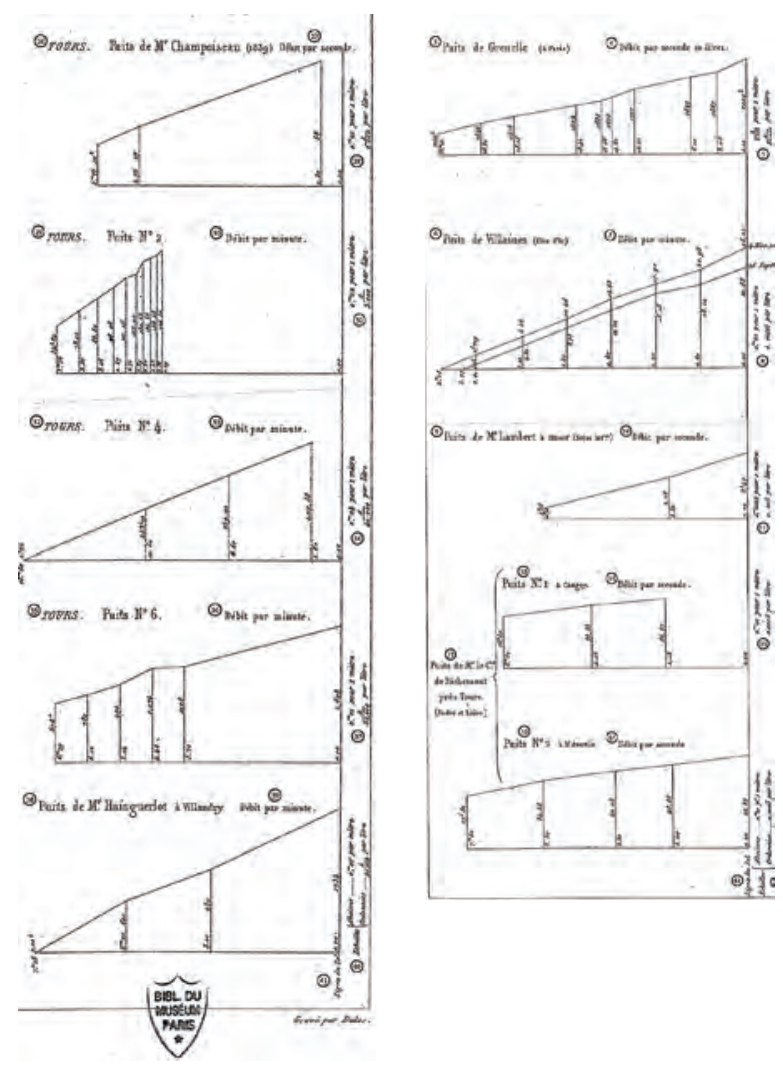

fig. 4 - Les débits des puits artésiens en fonction de la hauteur de l'orifice (extrait de Darcy Les fontaines publiques de la ville de Dijon, planche 22).

En guise de conclusion: une heuristique hypothétique En regroupant nos constatations, nous trouvons dans les deux livres de Darcy une théorie locale qui se présente de la façon suivante. La loi des écoulements lents dans les conduites est purement expérimentale. Elle sert de fondement théorique à la loi de Darcy. Les lois qui régissent l'abaissement du niveau de l'eau au-dessus d'une couche de sable et le rapport entre le débit d'un puits artésien et la hauteur de son déversoir se déduisent mathématiquement de la loi de Darcy (pourvu, pour la seconde, qu'on puisse négliger la perte de charge dans le tube ascensionnel). Chacune de ces quatre lois est vérifiée par l'expérience. Cependant plusieurs passages montrent que l'auteur les considère comme prouvées mais qu'il hésite entre la preuve expérimentale et la preuve par la cohésion de l'ensemble. Par exemple, la vérification de la loi de l'abaissement de la hauteur est invoquée pour valider la loi de Darcy dont elle dérive.

Un point faible évident (pour nous) de cet ensemble est l'extrapolation de la loi des écoulements lents des tuyaux au sable. Pour Darcy, elle paraît toute naturelle. Jetons un coup d'œil rétrospectif sur la question. II est facile de déduire la loi de Poiseuille des équations de Navier-Stokes ${ }^{55}$, mais cela n'a été fait que très $\operatorname{tard}^{56}$. L'histoire des déductions théoriques de la loi de Darcy n'a pas été faite, contentons-nous de quelques repères. Dupuit a cru la déduire de celle de Poiseuille en assimilant le sable à un assemblage de tubes très fins et très courts auxquels s'applique la loi de Poiseuille ... qui ne s'applique pas aux tubes courts.

On trouve un raisonnement analogue dans le livre de Jacob Bear, un ouvrage de référence ${ }^{57}$. Là, les tubes ne sont plus trop courts, mais nous sommes très loin de la structure du sable. II a fallu attendre la fin des années 1970 pour disposer d'une déduction convaincante par une méthode d'homogénéisation ${ }^{58}$.

Revenons à Darcy et, maintenant que nous avons synthétisé ses résultats, cherchons par quel chemin il a pu y arriver. Nous avancerons ici l'hypothèse que la découverte de la loi concernant les puits artésiens est antérieure à tout le reste et qu'elle a intrigué son auteur. Nous avons vu que lorsque Darcy décide de faire des expériences complémentaires pour vérifier que la perte de charge est proportionnelle au débit pour les écoulements lents dans les conduites, il ne dispose que de données expérimentales des plus minces pour étayer cette proportionnalité. De plus la question était extérieure au but des expériences. II a fallu qu'il soit sensibilisé à la recherche d'un tel rapport dans les écoulements pour y prêter attention.

55 Les équations de Navier-Stokes sont les équations aux dérivées partielles fondamentales de la mécanique des fluides. II n'est pas nécessaire de les écrire ici. Au temps de Darcy elles sont connues mais contestées et pas utilisées (O. Darrigol, Between hydrodynamics and elasticity theory, op. cit.). La loi de Poiseville (1841), qui se trouve déjà dans Hagen (op. cit.) a été rappelée plus haut dans une citation de Darcy. Sa déduction à partir des équations de NavierStokes permet de préciser la valeur de la constante $K: \pi / 128$ $\checkmark$ ( $v$ est la viscosité cinématique).

56 O. Darrigol, Worlds of flows, op. cit., p. 143.

57 Jacob Bear, Dynamics of Fluids in porous Media, New York, American Elsevier, 1972.

58 Les méthodes d'homogénéisation permettent de trouver des solutions approchées pour des problèmes sur des "grands " domaines présentant des inhomogénéités de petite taille, typiquement une plaque percée de trous petits et serrés. Un point clef, si u est une fonction inconnue définie sur le grand domaine, est de la mettre sous la forme :

$$
u(x)=v(x, x / \varepsilon)
$$

où $\varepsilon$, qui tend vers zéro, est une taille typique des inhomogénéités, et de calculer le premier terme non nul d'un développement en puissances de $\varepsilon$. Dans le cas de la loi de Darcy, $\varepsilon$ est une taille moyenne des pores ou des grains de sable. 